Research Article

\title{
High Fluorescence Lymphocyte Count and Immature Platelet Fraction; "Can these Two Parameters Reliably Distinguish between Dengue and ITP?"
}

\author{
Jawad Hassan ${ }^{*}$, , Samra Waheed ${ }^{1}$, Madiha Abid², Tahir Sultan Shamsi ${ }^{3}$ \\ ${ }^{1}$ Department of Diagnostic Hematology, National Institute of Blood Disease and Bone Marrow Transplantation, Karachi, Pakistan. \\ ${ }^{2}$ Research Department, National Institute of Blood Disease and Bone Marrow Transplantation, Karachi, Pakistan. \\ ${ }^{3}$ Department of Clinical Hematology, National Institute of Blood Disease and Bone Marrow Transplantation, Karachi, Pakistan.
}

\begin{abstract}
Objective: Thrombocytopenia is a frequent finding in Dengue fever. Immune Thrombocytopenia (ITP) is a diagnosis of exclusion and is not associated with febrile illness. Ignoring fever, low platelet count and clinical signs may be similar in both conditions. Peripheral film finding in Dengue fever shows many reactive lymphocytes. Enumeration of these reactive lymphocytes as high fluorescence lymphocyte count (HFLC) and computing immature fraction of platelets (IPF) may be useful in differentiating these two disorders as soon as the blood sample is analyzed on XN-1000 hematology analyzer.
\end{abstract}

Materials and Methods: A cross-sectional study was conducted at National Institute of Blood Disease and Bone Marrow transplantation from January to July 2019 during (Dengue season); blood samples from emergency room were analyzed on XN-1000 hematology analyzer for complete blood count and IPF. Samples with thrombocytopenia were checked for IPF count and HFLC from the extended research parameter data of the analyzer. Patients presenting with fever, had Dengue NS-1 tested. Detailed history \& examination was recorded from the patients.

Results: Out of the 124 patients, 62 (50\%) patients of Dengue and ITP respectively. Male to female ratio was 2:1. Mean age in Dengue was $25.52 \pm 10.46$ years while $34.44+20.82$ years in ITP group. Mean platelet count was significantly higher in dengue than ITP patients (120.59 $\pm 80.28 \times 10^{9} / \mathrm{L}$ versus $\left.41.84 \pm 38.62 \times 10^{9} / \mathrm{L}\right)(\mathrm{p}<0.001)$. HFLC was $11.71 \pm 7.17 \%$ in Dengue fever while $0.198 \pm 0.25 \%$ in ITP patients whereas IPF in ITP group was $21.91 \pm 16.09 \%$ while $8.79 \pm 4.39 \%$ in Dengue patients were found to be significant i.e. $(p<0.001)$.

Conclusion: Excluding fever, thrombocytopenia, presence of increased number of HFLC and low or normal IPF can reliably a predictor of a diagnosis in Dengue season.

Keywords: High fluorescence lymphocyte counts, Immature platelet functions, Dengue, Immune thrombocytopenia, Resource constraint Laboratories, Research parameters.

\section{INTRODUCTION}

Dengue fever has become one of the most common causes of undifferentiated fever in tropical settings; and is now among most periodically spreading mosquito borne disease in Pakistan along with malaria $[1,2]$. The cause of dengue fever is the single stranded enveloped RNA virus belonging to the genus Flavivirus. It is transferred to humans via bite of infected female mosquito of the genus Aedes, mostly Aedes aegypti and rarely Aedes albopictus. Four serotypes of dengue virus (DEN-1, DEN-2, DEN3, DEN-4) are yet identified and infection by any one serotype engender life-long immunity against that serotype, but develop partial protection against the other serotypes that can result in reinfection $[3,4]$. Subsequent infection with another serotype could be more severe and can lead to Dengue Hemorrhagic Fever [5].

The global incidence of dengue has developed persuasively in recent decades. A study estimated that there are around 390 million dengue virus infections annually [6]. According to the

*Address correspondence to this author at the Department of Diagnostic Hematology, National Institute of Blood Disease and Bone Marrow Transplantation, Karachi, Pakistan. Email: jawadkazmi2003@gmail.com recent data by World Health Organization, risk of dengue infection is present in 129 countries around the globe but $70 \%$ of the actual burden is possessed by Asia and dengue fever has been declared as an endemic in the subcontinent [7].

Dengue is a severe, flu-like illness that affects people of all ages, but seldom causes death (mortality is around $1 \%$ ). Symptoms usually last for 2-7 days, after an incubation period of 4-10 days. Typical dengue infection is characterized by high grade fever, severe headache, myalgia, arthralgia, retro-orbital pain and maculopapular rash $[8,9]$.

Several methods can be used for diagnosis of Dengue infection including virological tests (that directly detect elements of the virus) and serological tests, which detect human-derived immune components that are produced in response to the virus [10-12]. Patient samples collected during the first week of illness should be tested by both serological and virological methods according to the guidelines, however, in resource limited countries like Pakistan, it is very difficult to indulge huge amount of financial burden on the patient's diagnosis. It is therefore much needed to do appropriate 
testing on the basis of clinical details along with supportive lab parameters that can point towards definitive diagnostic investigation.

In around $50 \%$ of dengue fever infection, complete blood counts show thrombocytopenia [13]. Decreased platelet count has been associated with a large number of infectious and non-infectious causes and has to be differentiated on clinical and laboratory parameters. One of the causes of thrombocytopenia is Immune Thrombocytopenia (ITP) that manifests as a clinical syndrome of increased bleeding tendency, purpura and petechiae. Major cause of thrombocytopenia in ITP is due to the formation of antibodies against platelets that causes accelerated platelet destruction as well as impaired platelet production. Apart from complete blood count (CBC), new parameters on automated hematology analyzers are under research to differentiate between variant causes of thrombocytopenia. One such parameter is high-fluorescence lymphocytes count (HFLC). It indicates the presence of highly activated lymphocytes, such as activated B cells or plasma cells, which are increased during viral infections [14, 15]. Another parameter is immature platelet fraction (IPF) that measures the immature/young platelets present in peripheral blood and can indicate the degree of destruction and production of platelets by bone marrow [16].

Peripheral laboratories which receive samples from referral clinics and which do not have access to patient's clinical history or where there is no experienced pathologist for reporting peripheral blood film morphology, may result in missing the important finding related to patient's disease. It may end up in delaying patient's treatment. On the contrary, isolated thrombocytopenia without proper morphological findings may end up in unnecessary investigations, which may result in financial loss to the patient.

The purpose of this study is to determine whether these two parameters (HFLC and IPF) can reliably differentiate between Dengue fever and Immune Thrombocytopenia before carrying out further investigations.

\section{MATERIALS AND METHODS}

In this cross-sectional study; a total of 124 patients were included in the study, 62 patients each of dengue fever and Immune thrombocytopenia diagnosed between January to July 2019. Samples were collected from inpatient, outpatient and emergency department of National Institute of Blood disease and Bone marrow transplantation during this period. Di potassium ethylene di amine tetra acetic acid (EDTA) tubes were used for performing Complete blood count. Peripheral smear along with CBC, HFLC and IPF analysis were performed within 3 hours after blood collection on Sysmex XN 1000. HFLCs were analyzed in the WDF channel of the analyzer using polymethine fluorescent dye, with analysis based on the flow-cytometry principle whereas IPF determination used proprietary fluorescent dye containing polymethine and oxazine. IPF and HFLC values were reported in percentage form, normal range of IPF was considered to be $1-7 \%$ and of HFLC was $0-1.4 \%[17,18]$.

All patients who were placed in dengue subgroup were dengue NS1 antigen positive by rapid antigen detection through immunochromatographic method. Patients in the ITP group were diagnosed on the basis of clinical details and examination in conjunction with laboratory parameters. In the ITP subgroup, cutoff value of Platelet count was $<100 \times 10^{9} / \mathrm{L}$ as per guidelines [19]. Diagnosis of ITP was established according to the recent guidelines and cases of both Primary and Secondary ITP were included in the study [19]. Autoimmune profile, viral markers (Anti HCV, Anti HIV) and H-pylori antigen was tested in each of ITP patients. Patient of every age and gender were included in our study. Patient having any other infection or unusual laboratory parameter (deranged Renal or liver function tests in ITP group) were excluded.

\section{STATISTICAL ANALYSIS}

In this Retrospective observational study, statistical analysis was done by utilization of qualitative as well as quantitative variables in both ITP and dengue groups. Frequency was computed on qualitative variables such as gender and clinical symptoms while quantitative variables such as age, platelets, count, IPF and HFLC were determined by mean and standard deviation. Independent t-test and Pearson correlation was applied to assess the association between IPF and HFLC in dengue and ITP patients. A p-value $\leq 0.05$ are statistically significant. All data was entered and analyzed by using SPSS version 23 .

\section{RESULTS}

Out of 124 patients, $41(66.1 \%)$ were males and $21(33.8 \%)$ were female in the dengue subgroup. Whereas, in ITP patients $40(64.5 \%)$ males and $22(35.4 \%)$ females were observed. Mean age was $25.52 \pm 10.46$ years and $34.44 \pm 20.82$ years in dengue and ITP groups respectively. Mean platelet count was significantly higher in dengue than ITP patients (120.59 \pm $80.28 \times 10^{9} / \mathrm{L}$ versus $\left.41.84 \pm 38.62 \times 10^{9} / \mathrm{L}\right)(\mathrm{p}<0.001)$. Almost all $(97 \%)$ of dengue patients presented with fever whereas epistaxis was the major complaint of ITP patients. Clinical manifestations of dengue and ITP patients are shown in Fig. (1).

Mean IPF was lower in patients with dengue as compared to the ITP patients whereas mean HFLC values were much higher in the dengue patients as compared to ITP patients, and association of both variables were found to be statistically 
significant $(\mathrm{p}<0.001)$ as shown in Table $\mathbf{1}$.

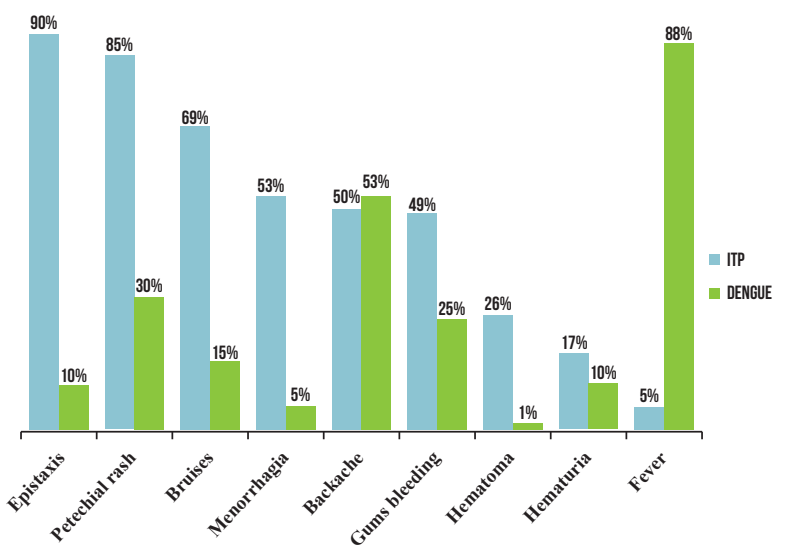

Fig. (1). Clinical Manifestation of Dengue and ITP Patients $(n=124)$.

Table 1. Association of HFLC and IPF\% in Dengue and ITP Group ( $\mathrm{n}=124)$.

\begin{tabular}{|l|l|l|l|}
\hline Disease & \multicolumn{1}{|c|}{$\begin{array}{c}\text { Dengue } \\
(\text { Mean } \pm \text { SD })\end{array}$} & $\begin{array}{c}\text { ITP } \\
(\text { Mean } \pm \text { SD) }\end{array}$ & p-Value \\
\hline$*$ IPF \% & $8.79 \pm 4.39$ & $21.91 \pm 16.09$ & $<0.001$ \\
\hline$* *$ HFLC\% & $11.71 \pm 7.17$ & $0.198 \pm 0.259$ & $<0.001$ \\
\hline
\end{tabular}

*IPF: Immature Platelet Fractions, **HFLC: High Fluorescence Liquid Chromatography.

On the other hand, when we correlated HFLC and IPF through Pearson correlation coefficient in dengue positive patients, a weak positive correlation was observed with ( $\mathrm{r}$ $=0.212 \& \mathrm{p}=0.098$ ) where as in ITP patients a very weak negative correlation was observed $(r=-0.059$ and $\mathrm{p}=0.647$ ) and both the correlations were statistically insignificant.

\section{DISCUSSION}

Definitive diagnosis of dengue fever requires antigen or antibody testing as per number of the days involved, however, due to overlapping features between dengue fever and fever due to other infectious causes, supporting laboratory investigations are required before jumping on to numerous expensive investigations [20, 21]. High Fluorescence Lymphocyte Count (HFLC) is considered to be an important tool in cases of dengue fever [22]. HFLC basically detects atypical lymphocytes present in the blood of dengue patient. Atypical lymphocytes are detected by their characteristically high fluorescence intensity reflecting a high RNA content [23]. Our study suggested that high HFLC \% is observed in dengue patients and can be used as an effective screening tool. These findings coincides with a previous study comparing dengue fever with other infectious diseases including typhoid, leptospirosis. Conclusion of that study showed that increased
HFLC (AUC of 0.89 with 95\% CI) along with increased number of atypical lymphocytes in the peripheral film were suggestive of dengue viral infection [24, 25]. Similarly, in dengue fever patients, increased numbers of atypical lymphocytes along with their flow cytometric analysis is also reported in few other studies however, their association with HFLC was not analyzed [26, 27].

Moreover, in our study, Immature Platelet Fraction (IPF) percentage was also measured in dengue patients and was estimated to be higher than the normal values (1-7\%) [17], however, it was found out to be significantly lower than the ITP patients ( $p$-value $<0.001$ ). This finding also corresponds to the previous study in which IPF was found to be higher in dengue patients (AUC of 0.81 with 95\% CI) [24]. However, we could not find any study comparing the IPF percentage of dengue with ITP patients.

Tantanate C, et al. reported that HFLC was found to be highest in group of patients having infectious disease (majority had dengue) and was lower in other disease groups such as immunological disorders and malignancies [25]. It coincides with the findings of our study that demonstrated lower HFLC values in ITP patients. Another significant finding in this study was significantly high IPF values in ITP patients and many studies have been conducted and have revealed the importance of IPF in ITP patients [28-30]. IPF is now a reportable parameter in ITP patients and not only helps the clinician in diagnosing ITP but also minimizing the unnecessary and invasive investigations such as bone marrow biopsy especially in young adults. In one study IPF was found to be $>7 \%$ in $85 \%$ of ITP patients [17].

Fever was the most common presenting complaint of dengue patients in our study which is in accordance with the previous studies published [31].

\section{Strength of Our Study}

1) It is the only study comparing HFLC and IPF parameters together.

2) Both parameters were not previously compared simultaneously in dengue and ITP.

3) Equal number of patients in both disease groups.

4) All dengue patients were analyzed at the time of symptom onset and not during the convalescent phase.

\section{Limitations to Our Study}

1) Small sample size, due to which the correlation established was weak.

2) We did not classify dengue fever patients into different risk groups so could not identify HFLC significance in establishing severity of the disease.

3) HFLC is a research parameter and established reference 
ranges are not available for both IPF and HFLC due to scarce literature.

\section{CONCLUSION}

Dengue fever patients have significantly increased HFLC values when compared to ITP patients; however, IPF is increased significantly in ITP patients more than dengue fever. On the basis of above mentioned findings, it can be concluded that dengue fever can be differentiated from ITP on the basis of HFLC and IPF parameters. It will not only help in reducing the financial burden on the patient but also help the clinician to commence only necessary testing. Moreover, it will also reduce the burden of reviewing all the slides and would be time saving for a pathologist. Also in resource limited peripheral labs, these parameters will help identify important information. Limited data for the use of HFLC in the diagnosis is yet available; however, its utilization could be really helpful in dengue endemic resource constraint countries. Lastly we would suggest the addition of HFLC in the list of routine reportable parameters after evaluating its significance and establishing the reference ranges in large randomized control trials.

\section{AUTHORS' CONTRIBUTION}

Jawad Hassan: Did study design, data interpretation, literature search, manuscript writing.

Samra Waheed: Preparing figures/tables, data interpretation and review manuscript.

Madiha Abid: Did statistical analysis.

Tahir Sultan Shamsi: Involved in study design, and supervision throughout the study.

\section{CONFLICT OF INTEREST}

Declared none.

\section{ACKNOWLEDGEMENTS}

Declared none.

\section{REFERENCES}

[1] Dengue guidelines for diagnosis, treatment, prevention and control. New edition. Geneva: WHO and TDR 2009; Available at: https://www.who.int/tdr/publications/documents/dengue-diagnosis.pdf

[2] Sherin A. Dengue fever: A major public health concern in Pakistan. KMUJ 2011; 3(1): 1-3.

[3] Halstead SB. Pathogenesis of dengue: Challenges to molecular biology. Science 1988; 239: 476-81. DOI: 10.1126/science. 3277268

[4] Kurane I. Dengue haemorrhagic fever with special emphasis on immunopathogenesis. Comp Immunol Microbiol Infect Dis 2007; 30: 329-40. DOI: 10.1016/j.cimid.2007.05.010
[5] WHO. Comprehensive guidelines for prevention and control of dengue and dengue haemorrhagic fever. $2^{\text {nd }}$ ed. Geneva: World Health Organization 2011.

[6] Bhatt S, Gething PW, Brady OJ, et al. The global distribution and burden of dengue. Nature 2013; 496(7446): 504-7. DOI: 10.1038 /nature 12060

[7] WHO. Dengue and Severe Dengue classification, news room. 2020; Available at: https://www.who.int/news-room/q-a-detail/dengue-and-severe-dengue

[8] Halstead SB. Dengue and dengue haemorrhagic fever. Clin Microbiol Rev 1998; 1: 480-96. DOI: 10.1128/CMR.11.3.480

[9] Munir MA, Alam SE, Uddin Khan Z, et al. Dengue fever in patients admitted in tertiary care hospitals in Pakistan. JPMA 2014; 64(5): 553-4.

[10] Irshad S, Saeed A. Dengue fever in Pakistan: Current updates. IJAVMS 2012; 6(1): 14-26. DOI: 10.5455/ijavms.165

[11] Lanciotti RS, Calisher CH, Gubler DJ, Chang GJ, Vorndam AV. Rapid detection and typing of dengue viruses from clinical samples by using reverse transcriptase-polymerase chain reaction. JCM 1992; 30(3): 545551. DOI: 10.1128/jcm.30.3.545-551.1992

[12] Parkash O, Shueb RH. Diagnosis of dengue infection using conventional and biosensor based techniques. Viruses 2015; 7(10): 5410-27. DOI: 10.3390/v7102877

[13] Azeredo EL de, Monteiro RQ, De-Oliveira pinto LM. Mint: Thrombocytopenia in dengue: Interrelationship between virus and the imbalance between coagulation and fibrinolysis and inflammatory mediators. Mediat Inflamm 2015; 3: 138-42. DOI: $10.1155 / 2015 / 313842$

[14] Linssen J, Jennissen V, Hildmann J, et al. Identification and quantification of high fluorescence-stained lymphocytes as antibody synthesizing/secreting cells using the automated routine hematology analyzer XE-2100. Cytometry B Clin Cytom 2007; 72(3): 157-66. DOI: 10.1002/cyto.b.20150

[15] Van Mirre E, Vrielink GJ, Tjon-a-Tsoi N, Hendriks H, de Kieviet W, ten Boekel E. Sensitivity and specificity of the high fluorescent lymphocyte count-gate on the Sysmex XE-5000 hematology analyzer for detection of peripheral plasma cells. Clin Chem Lab Med 2011; 49(4): 685-8. DOI: 10.1515/CCLM.2011.100

[16] Briggs C, Harrison P, Grant D, Staves J, Chavda N, Machin SJ. New quantative parameters on a recently introduced automated blood cell counter - the XE-2100. Clin Lab Haematol 2000; 22: 345-50. DOI: 10.1046/j.1365-2257.2000.00330.x

[17] Naz A, Mukry SN, Shaikh MR, Bukhari AR, Shamsi TS. Importance of immature platelet fraction as predictor of immune thrombocytopenic purpura. Pak J Med Sci 2016; 32(3): 575-9. DOI: $10.12669 /$ pjms.323.9456 
[18] Raharjo B, Hadi S. High fluorescent lymphocyte count examination in dengue hemorrhagic patients with SYSMEX Xn-1000 hematology analyzer. Indonesian J Clin Pathol Med Lab 2019; 25 (2): 207 -10. DOI: 10.24293/ijcpml.v25i2.1443

[19] Neunert C, Terrell Dr, Arnold DM, et al. American Society of Hematology 2019 guidelines for immune thrombocytopenia, blood advances. Blood Adv 2019; 3(23): 3829-66. DOI: 10.1182/bloodadvances.2019000966

[20] Simmons CP, Farrar JJ, Nguyen V, Wills B. Dengue. N Engl J Med 2012; 366: 1423-32. DOI: 10.1056/NEJMra1110265

[21] Libraty DH, Myint KS, Murray CK, et al. A comparative study of leptospirosis and dengue in Thai children. PLoS Negl Trop Dis 2007; 1: e111. DOI: 10.1371/journal.pntd.0000111

[22] Briggs CJ, Linssen J, Longair I, Machin SJ. Improved flagging rates on the Sysmex XE-5000 compared with the XE-2100 reduce the number of manual film reviews and increase laboratory productivity. Am J Clin Pathol 2011; 136: 309-16. DOI: 10.1309/AJCPDLR4KGKAFW4W

[23] Zini G, Bain B, Bettelheim P, et al. A European consensus report on blood cell identification: Terminology utilized and morphological diagnosis concordance among 28 experts from 17 countries within the European Leukemia Net network WP10, on behalf of the ELN Morphology Faculty. Br J Haematol 2010; 151: 359-64. DOI: 10.1111/j.1365-2141.2010.08366.x

[24] Oehadian A, Michels M, De Mast Q, et al. New parameters available on Sysmex XE-5000 hematology analyzers contribute to differentiating dengue from leptospirosis and enteric fever. Int J Lab Hematol 2015; 37: 861-8.
[25] Tantanate C, Klinbua C. Performance evaluation of high fluorescence lymphocyte count: comparability to atypical lymphocyte count and clinical significance. Lab Med 2018; 49; 362-8. DOI: 10.1093/labmed/lmy030

[26] Jampangern W, Vongthoung K, Jittmittraphap A, et al. Characterization of atypical lymphocytes and immunophenotypes of lymphocytes in patients with dengue virus infection. Asian Pac J Allergy Immunol 2007; 25(1): 27-36.

[27] Cui W, Wu Y, Xie H. Correlation between the cell population in the automated hematology analyzer high-fluorescence region and atypical lymphocyte flags. J Clin Lab Anal 2018; 32: e22374. DOI: $10.1002 /$ jcla.22374

[28] Briggs C, Kunka S, Hart D, Oguni S, Machin SJ. Assessment of an immature platelet fraction (IPF) in peripheral thrombocytopenia. $\mathrm{Br} \mathrm{J}$ Haematol 2004; 126: 93-9. DOI: 10.1111/j.1365-2141.2004.04987.x

[29] Briggs C, Harrison P, Grant D, Staves J, Chavda N, Machin SJ. New quantative parameters on a recently introduced automated blood cell counter - the XE-2100. Clin Lab Haematol 2000; 22: 345-50.

DOI: $10.1046 / j .1365-2257.2000 .00330 . \mathrm{x}$

[30] Kickler TS, Oguni S, Borowitz MJ. A clinical evaluation of high fluorescent platelet fraction percentage in thrombocytopenia. Am J Clin Pathol 2006; 125(2): 282-7. DOI: 10.1309/50H8JYHN9JWCKAM7

[31] Guha-sapir D, Schimmer B. Dengue Fever: New paradigms for a changing epidemiology. Emerg Themes Epidemiol 2005; 2: 1. DOI: $10.1186 / 1742-7622-2-1$ 